\title{
Consolidation Treatment and Long-Term Prognosis of Rituximab in Minimal Change Disease and Focal Segmental Glomerular Sclerosis
}

\author{
Li Lin' \\ Weiming Wang' \\ YiFan $\mathrm{Wu}^{2}$ \\ JingYuan Xie' \\ Xiao $\mathrm{Li}^{1}$ \\ XiaoXia Pan' \\ Wen Zhang' \\ Jing $X u^{\prime}$ \\ YiKai Cai \\ Hong Ren' \\ Nan Chen'
}

'Department of Nephrology, Ruijin Hospital, School of Medicine, Shanghai Jiaotong University, Shanghai, People's Republic of China; ${ }^{2}$ Biomedical and Health Informatics, University of Washington, Seattle, WA, USA
Correspondence: Hong Ren; Nan Chen Department of Nephrology, Ruijin Hospital, School of Medicine, Shanghai Jiaotong University, No. 197, Ruijin Er Road, Shanghai, People's Republic of China

Tel +862164370045 , extension 665233

Fax +86 21 64456419

Email renhong66@I26.com;

cnrj100@I26.com
Purpose: There is currently a lack of studies investigating long-term prognosis and the necessity of further rituximab (RTX) consolidation treatment for minimal change disease (MCD) and focal segmental glomerulosclerosis (FSGS). The aim of this study was to evaluate the efficacy of RTX for these diseases and to investigate whether a consolidation treatment can lower risks of relapse and reinforce long-term remission.

Patients and Methods: A retrospective study was conducted. The relapse and remission of 70 patients treated with 1 course of RTX treatment ( 4 infusions of $375 \mathrm{mg} / \mathrm{m} 2$ ) over a median follow-up time of 27 months (12-60 months) were analyzed. The rates of patients that were able to achieve non-relapse for a duration of 24 months between RTX consolidation therapy and non-consolidation therapy were compared.

Results: There were 67 cases $(95.71 \%)$ of remission and 3 cases $(4.29 \%)$ of non-remission. The average number of relapses decreased from $3.7 \pm 2.5$ times before the treatment to 0.8 \pm 1.8 times after treatment $(\mathrm{P}<0.001)$. The average avannual number of relapses decreased from $1.3 \pm 1.2$ times/year to $0.2 \pm 0.3$ times/year $(\mathrm{P}<0.001)$. The results from the Cox proportional-hazards model showed that the risk of relapse in patients who received RTX nonconsolidation treatment was significantly higher than those with consolidation treatment (odds ratios (OR) 20.9, 95\% confidence intervals (CI) OR 5.7-75.7, p<0.001). The 24month relapse-free rate was also significantly higher in patients with consolidation therapy compared with non-consolidation therapy $(86.36 \%$ vs $25 \%$, p $<0.001)$. No adverse events were recorded.

Conclusion: RTX is highly effective in treating MCD and FSGS, and RTX consolidation therapy may be recommended to reinforce long-term remissions.

Keywords: RTX, MCD, FSGS, consolidation, therapeutic effect

\section{Introduction}

Minimal change disease (MCD) and focal segmental glomerulosclerosis (FSGS) account for $60 \%$ of nephrotic syndrome (NS) - one of the main causes of glomerulosclerosis in China. ${ }^{1}$ RTX which is a CD20 lymphocyte monoclonal antibody that causes B lymphocyte depletion ${ }^{2}$ is widely used in treatments of autoimmune diseases and immune-mediated kidney diseases such as lupus erythematosus and vasculitis. $^{3,4}$ Depletion of B cells that interact with T cells in MCD, and participate in the production of circulating factors in FSGS, is achieved through antiproteinuric effects of RTX through methods of direct, indirect, and cytotoxic effects. In addition to modifying effects on $\mathrm{T}$ cell subsets, RTX also depletes a portion of 
T cells that coexpress both $\mathrm{CD} 3$ and $\mathrm{CD} 20 .^{5}$ In recent years, it has been reported to successfully treat refractory NS. ${ }^{6-10}$ Gilbert et al first reported the use of RTX in the treatment of children with steroid-dependent NS. Subsequent studies have further verified that RTX can reduce the likelihoods of relapse when steroids and other immunosuppressive drugs are withdrawn for children with MCD. ${ }^{11}$

Previously, our team has also reported remission in fifteen FSGS patients after RTX was administered. These patients had exhausted options such as steroid and immunosuppressant agents. ${ }^{12}$ To this date, although there are no randomized controlled trials (RCTs) demonstrating the efficacy of RTX in FSGS/MCD patients, observational studies have confirmed its efficacy. ${ }^{13}$ Yet RTX is not included as a first-line treatment for MCD and FSGS. Current recommended first-line treatments for MCD and FSGS are limited to a high-dose steroid, cyclophosphamide (CTX), calcineurin inhibitors (CNI), or mycophenolate Mofetil (MMF), with steroid therapies being the most conventional. Reported statistics for the sensitivity of steroids were $75 \%$ for MCD and $20-60 \%$ for FSGS, ${ }^{14-16}$ however, approximately $40 \%$ of steroid-sensitive patients may experience relapse after steroid reduction or withdrawal. These cases then require further treatment consisting of sufficient or medium-dose steroids combined with immunosuppressive therapy. ${ }^{15,16}$ Potential side effects may include infection, diabetes, osteoporosis, osteonecrosis of the femoral head, gonadal inhibition, myelosuppression, and obesity. ${ }^{1}$

In light of these factors, RTX looks to be an alternative as we did not observe negative effects in our patients after its administration. This study aims to critically assess our past success through retrospective analysis. By investigating the efficacy of RTX in the long term and whether consolidation therapy can further lower the relapse rate, we hope to illuminate the viability and necessity of consolidation treatment for MCD and FSGS patients.

\section{Patients and Methods}

This is a retrospective cohort study on the long-term efficacy of RTX in the treatment of MCD and FSGS that also explores the benefits of additional consolidating treatment for remission patients. All patients received one course of RTX treatment (4 infusions of $375 \mathrm{mg} / \mathrm{m} 2$, every 7-14 days). Optional courses of RTX consolidation (1-2 infusions of $375 \mathrm{mg} / \mathrm{m} 2$ ) were then given 6 months after patients were able to achieve and maintain remission.
Written informed consent was obtained from all patients. This research was approved and in accordance with the ethics committee of Ruijin Hospital affiliated with Jiao Tong University (Approval Document No. 219, 2019), and with the 1964 Helsinki declaration and its later amendments.

\section{Study Patients}

Adult patients enrolled in the study were those with renal biopsy-proven FSGS and MCD, eGFR higher than $30 \mathrm{~mL} /$ min as calculated by the EPI equation, and had completed 1 course of RTX treatment (4 infusions of $375 \mathrm{mg} / \mathrm{m} 2$ ) at Shanghai Ruijin Hospital from June 2014 to June 2019. Steroids and immunosuppressants were gradually reduced and ultimately withdrawn within 3 months after receiving RTX treatment in remission patients, however orally taken renin-angiotensin system (RAS) inhibitors were not halted. Patients who had hereditary nephropathy, pregnancy, tumor, and glomerulonephritis other than FSGS or MCD were excluded from the study. Follow-up duration was no less than 12 months for all remission patients.

\section{Study Procedures}

Upon enrollment, data were collected for the baseline, every 3 months after RTX use, at relapse, and last followup. Information collected included demographics, blood pressure, side effects, routine blood, and urine tests for liver and renal function consisting of 24-hour proteinuria, serum glucose, serum immunoglobulins (IgG, IgM, IgA, IgE), serum CD19+ and CD20+ leukomonocytes. Before RTX use, conventional screenings that were done include HBV-DNA, HBV-Cobas, HCV-Cobas, RPR, HSV-DNA, CMV-DNA, T-Sport, sputum, midstream urine bacterial and fungal cultures, and chest CT.

For this study, the definition of consolidation therapy was additional RTX therapy given to patients that achieved and maintained 6 months of partial remission (PR) or complete remission (CR) after the first round of RTX treatment. The definition of control group (nonconsolidation) were patients who did not receive consolidation treatment. A relapse was defined as an increase of protein excretion $>3.5 \mathrm{~g} / \mathrm{d}$; time to relapse was calculated from the time of therapy initiation to onset of relapse; CR was defined as a $24 \mathrm{~h}$ urine protein excretion $<0.3 \mathrm{~g} / \mathrm{d}$; PR was defined as $>50 \%$ reduction of proteinuria from baseline; non-remission (NR) was defined as $<50 \%$ reduction compared to the peak of proteinuria within 6 months from therapy initiation; time to $\mathrm{PR}$ or $\mathrm{CR}$ was described as the 
time from therapy initiation to the first day that $\mathrm{PR}$ or $\mathrm{CR}$ was observed; B cell depletion was defined as CD19+lymphocyte count $<5$ cells/ul; B-cell recovery was defined by a CD19+ lymphocyte count of $>15$ cells/ul; annual number of relapses before RTX treatment equals the total number of relapses divided by total disease duration using year as unit; annual number of relapses after RTX treatment equals the number of relapses divided by followup starting with the first dose of RTX using year as unit.

The remission ( $\mathrm{CR}$ or $\mathrm{PR})$ rate, relapse rate, and no remission rate at the last follow-up were analyzed to evaluate the overall efficacy of RTX treatment. The effect of RTX on reducing relapse was evaluated by comparing the number of relapses, the number of relapses in the first year and the year before, and the average annual number of relapses before and after RTX treatment. Subgroup analysis was performed on the patients who completed the 24-month follow-up to analyze the effects of consolidation therapy and non-consolidation therapy on the relapse-free rate by 24 months.

\section{Statistical Analysis}

The remission rate was analyzed using the $\chi^{2}$ test. KaplanMeier curves were used to visualize time to first relapse and graphically compare before and after RTX therapy, the consolidation treatment, and the control group. Time to first relapse was analyzed using a Cox proportionalhazards model. The factors associated with relapse in the univariate analysis $(\mathrm{P} \leq 0.20)$ were included in the multivariable analysis with a forward stepwise selection procedure. In the first step, all variables associated with relapse by univariate analysis $(\mathrm{P}<0.20)$ were entered into the model. Variables were dropped if they were no longer significant when adjusted with other variables. Odds ratios (ORs) and their 95\% confidence intervals (95\% CI) were computed. The significance level was set at $\mathrm{p}<0.05$. Statistical analyses were performed using SPSS version 20 (SPSS Inc., Chicago, IL, USA).

\section{Results}

\section{Patient Population}

Seventy patients fulfilled the selection criteria and were enrolled in the study from June 2014 to June 2019. The median age of the study population was 25 (19, 36 interquartile range IQR) years old and $35.7 \%$ were female. All 70 patients received 4 doses of $375 \mathrm{mg} / \mathrm{m}^{2} \mathrm{RTX}$ and all patients took RAS inhibitors, except for 2 patients whose blood pressure was too low.

Among the 70 patients, biopsies confirmed that 49 patients had MCD and 21 patients had FSGS. At baseline, all patients had severe nephrotic syndrome (NS), with a median disease duration of 36 months (ranging from 18 to 105 months, IQR (Table 1). Of the 70 patients, 67 failed prior steroid with/without immunosuppressive treatment, 7 never achieved remission from NS, 60 suffered from 3.7 \pm 2.5 number of relapses, and 3 patients did not receive any prior treatments (Table $\mathrm{S} 1)$.

The median of 24h-proteinuria in enrolled patients was $2.2 \mathrm{~g} / 24 \mathrm{~h}$ (ranging from $0.2-7.8$, IQR (Table 1). NS was present in 32 patients (46\%), of which 7 never achieved remission, 3 did not receive treatments, and 22 relapsed. The other 38 patients (54\%) with non-NS were treated with rituximab to reduce relapse and to discontinue steroid and immunosuppressant therapy.

Table I Main Clinical and Laboratory Characteristics at Baseline

\begin{tabular}{|c|c|}
\hline Characteristic $(n=70)$ & Value \\
\hline Age-years Median (IQR) & $25(19,36)$ \\
\hline Female sex-no. (\%) & $25(35.7)$ \\
\hline Blood pressure- $\mathrm{mm} \mathrm{Hg}$ & \\
\hline Systolic (Mean) & $127.0+16.4$ \\
\hline Diastolic (Mean) & $77.0 \pm 11.5$ \\
\hline Height-cm & $166.0 \pm 10.7$ \\
\hline Weight—kg & $65.0 \pm 16.0$ \\
\hline BMI & $23.0 \pm 5.2$ \\
\hline Number of relapses before RTX therapy & $3.7 \pm 2.5$ \\
\hline Disease duration before RTX therapy-months & \\
\hline Median (IQR) & $36(18,105)$ \\
\hline $\begin{array}{l}\text { History of steroid/immunosuppressive therapy-no. } \\
\text { (\%) }\end{array}$ & $67(95.7)$ \\
\hline Serum albumin—g/l & \\
\hline Median (IQR) & $28(16,36)$ \\
\hline Serum creatinine-umol/l & $81.0 \pm 47.6$ \\
\hline Urinary protein-g/24 hr & \\
\hline Average & 5.2 \\
\hline Median (IQR) & $2.2(0.2-7.8)$ \\
\hline Creatinine clearance- $\mathrm{mL} / \mathrm{min} / 1.73 \mathrm{~m}^{2}$ & $109.0 \pm 29.5$ \\
\hline
\end{tabular}

Abbreviations: BMI, body mass index (calculated as weight in kilograms divided by height in meters squared); IQR, interquartile range. 


\section{Response to Rituximab and Relapses}

After RTX treatment, all patients were in states of complete depletion for CD19. There were 67 cases (95.71\%) of remission [15 (21.43\%) PR, 51 (74.29\%) CR], and 3 (4.29\%) NR. For remission patients, the median follow-up time was 27 months (12-60 months) and median proteinuria at the last follow-up was $0.15 \mathrm{~g} / 24 \mathrm{~h}(0.09,0.41$, IQR). Steroids and immunosuppressants were gradually reduced and ultimately withdrawn within 3 months.

Of the 49 MCD patients, there were 47 cases $(95.92 \%)$ of remission [8 (16.33\%) PR, 39 (79.59\%) CR], 2 (4.08\%) $\mathrm{NR}$, and $33(70.21 \%)$ relapse-free. Median duration of sustained remission was 16 months (13, 36 months, IQR).

Of the 21 FSGS patients, 20 cases (95.24\%) experienced remission [7 (33.33\%) PR, 13 (61.90\%) CR], 1 $(4.76 \%) \mathrm{NR}$, and $13(65.00 \%)$ relapse-free. Median duration of sustained remission was 18 months $(12,27$ months, IQR).

Of the 32 NS patients, 29 cases (90.53\%) experienced remission [10 (31.24\%) PR, 19 (59.38\%) CR], 3 (9.38\%) $\mathrm{NR}$, and $22(68.75 \%)$ relapse-free. Median duration of sustained remission was 17 months (13, 24 months, IQR). Of the 38 non-NS patients, 24 (63.18\%) were relapse-free. Median duration of sustained remission was 33 months (18, 45 months, IQR).

Overall, 46 (65.71\%) cases were relapse-free, of which the median duration of sustained remission was 18 months (13, 35 months, IQR). 31 patients received RTX consolidation therapy. The average number of consolidation treatments was $1 \pm 0.7$, and the average interval time of consolidation treatment was $12 \pm 3$ months. Of the other 21 cases $(30.00 \%)$ that experienced relapse, all were able to re-achieve remission (100\%) after the second course of RTX treatment. The average number of relapses for these patients was $0.8 \pm 1.8$ times, and the average interval time of relapses was $11 \pm 4$ months.

\section{Comparison Before and After RTX Treatments}

After RTX treatment, the average number of relapses among patients decreased from $3.7 \pm 2.5$ times to $0.8 \pm 1.8$ times $(\mathrm{P}<0.01$, Figure S1A). The average number of relapses between the year before RTX treatment compared to the first year after treatment decreased from $0.8 \pm 0.6$ times to $0.2 \pm 0.3$ times $(\mathrm{P}<0.01$, Figure $\mathrm{S} 1 \mathrm{~B})$. The average annual number of relapses decreased from $1.3 \pm 1.2$ times/ year to $0.2 \pm 0.3$ times/year $(\mathrm{P}<0.01$, Figure 1$)$.

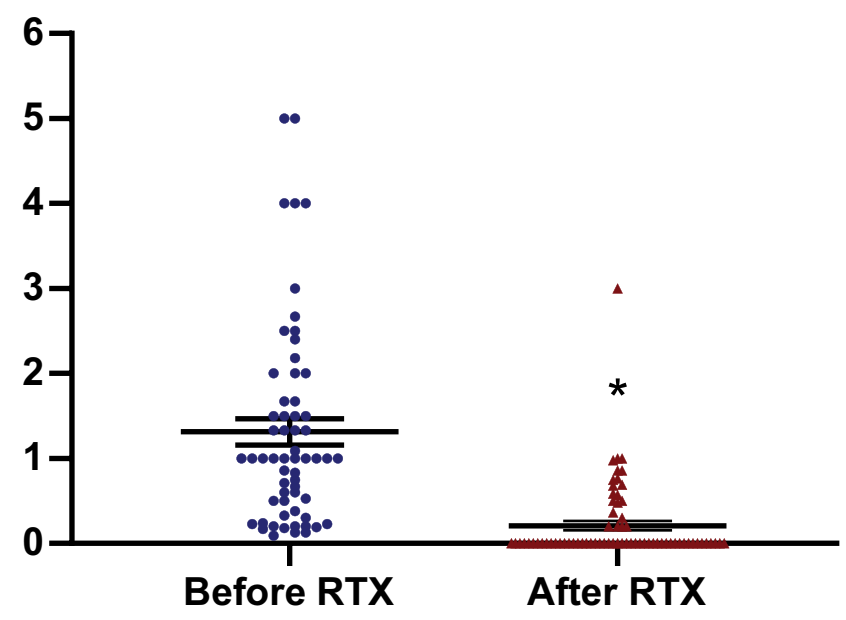

Figure I The number of relapses $\pm S D$ per year before and after rituximab treatment $(* \mathrm{P}<0.05)$.

For the 21 relapsed patients, the median time to first relapse was 12.5 months (11, 19.5 months, IQR), which is significantly longer than the median time to the first relapse before RTX treatment of 5.5 months (3, 7 months, IQR) $(<0.001)$ (Figure 2). Before RTX treatment, 37 patients $(61.67 \%)$ had more than 2 relapses. After RTX treatment, only 6 patients $(9.09 \%)$ had more than 2 relapses $(\mathrm{p}<0.001)$.

\section{Risk Factors for Relapse}

Patients who had previously been treated with multi-target $(\mathrm{p}=0.009)$, cyclophosphamide $(\mathrm{p}=0.003)$, and tacrolimus $(\mathrm{p}=0.019)$ were more likely to suffer relapses compared to the patients who did not receive these treatments. The risk of developing a relapse in patients who received RTX consolidation treatment was significantly lower than that in non-relapsed patients $(p<0.001)$. The follow-up time of the relapsed patients was longer, and the proportion of CD19 recovery in the relapsed patients was higher (Table 2).

A multivariate Cox regression analysis for relapse was performed using the treatment history of steroid, cyclophosphamide and tacrolimus, and cyclosporin. Other factors included were disease duration and relapse time before RTX use, CD19+ B-cells non-recovery, the use of consolidation treatment, and follow-up time after RTX use. The risk of relapse without consolidation treatment was 20.9 times higher than with consolidation (95\% CI: 5.7-75.7, $\mathrm{p}<0.001$ ), and 6.3 times higher for CD19+ B-cells recovery compared to CD19+ B-cells non-recovery $(95 \% \mathrm{CI}$ : 2.0-19.0 p =0.001). (Table 3, Figure S2) 


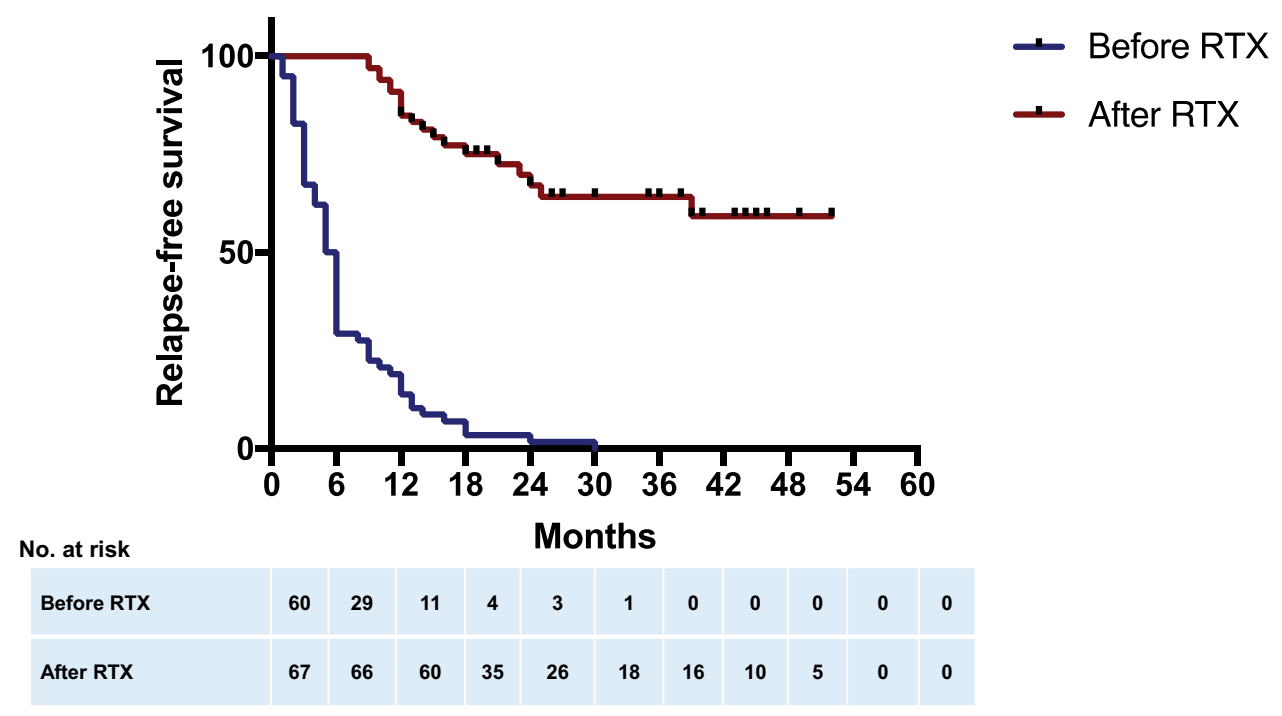

Figure 2 Relapse-free survival before and after RTX therapy in the overall cohort.

Notes: The relapse-free survival rate was significantly higher for patients after taking RTX therapy compared to before (log-rank $p<0.001)$. This graph details the amount of time for the percentage of patients to start relapsing. Both lines describe the same group of patients, and the red line follows the blue line in terms of time-frame. We can see that before using RTX (blue line), all patients relapsed before 30 months, while after using RTX (red line), more than $50 \%$ did not see relapse by the end of the 54 th month according to analysis.

\section{The Effect of Consolidation Therapy for 24-Month Relapse-Free Survival}

Forty-two patients who completed a 24-month follow-up were included. Of these, 22 patients were in the consolidation group, and 20 patients were in the non-consolidation group. There were no statistical differences in age, gender, pathology, duration, previous drug treatment (tacrolimus being the exception), $24 \mathrm{~h}$ proteinuria, blood albumin, creatinine at baseline between the two groups (Table 4).

The percentage of patients that remained relapse-free for 24 months after consolidation treatment (19 cases $(86.36 \%)$ ) and non-consolidation treatment (5 cases, $(25 \%))$ were significantly different $(\mathrm{p}<0.001)$. The median relapse time was 24 months for consolidation treatment and 14 months for non-consolidation treatment $(\mathrm{p}<0.001)$. Figure 3 illustrates the differences in time to the first relapse between the two groups.

\section{Adverse Events}

RTX infusion could sometimes lead to low fever, itching of the scalp and skin, and rash. All six patients that experienced a combination of these symptoms were relieved after applying slow infusion. During follow-up, six patients developed skin allergies. After administering antiallergic symptomatic treatments, all symptoms disappeared within 6 months after RTX treatment. Six patients had an abnormal liver function, which returned to normal after receiving liver protection treatments.

\section{Discussion}

Although RTX is not yet recommended as a first-line treatment for MCD and FSGS, it has shown promising results in this study and several previous studies. For FSGS and MCD, our results demonstrate a remission rate of $95.71 \%$ with $74.29 \% \mathrm{CR}, 21.43 \% \mathrm{PR}$, and $4.29 \% \mathrm{NR}$, and the relapse rate was $30.00 \%$. These results are better in comparison to the total remission rate of $67 \%{ }^{17}$ achieved by steroids or/and immunosuppression in treating FSGS and nephrotic range proteinuria from previous studies. Most studies also reported that RTX performs better than calcineurin inhibitors for patients with refractory NS. ${ }^{6-9,11,18-26}$ These statistics suggest that RTX is on par or more effective than some of the existing recommended first-line options. A recent meta-analysis also reported the overall remission and relapse of RTX therapy of $53.6 \%$ and $47.3 \%$, respectively in FSGS and $80.3 \%$ and $35.9 \%$ in MCD. ${ }^{27}$ The higher remission rate of this study compared to previous reports is likely related to ethnic differences. In addition, $54 \%$ of patients have achieved remission through steroid and immunosuppressant therapy, thus the use of RTX was solely to reduce relapse and to discontinue other drug use. Pathological typing distribution could be another factor. At our center, for FSGS, NOS (No-specific) was of highest prevalence $(73.6 \%)$, followed by perihilar (14.1\%), tip (9.2\%), and collapsing variants (2.5\%). Collapsing variants commonly seen in African Americans were studied in other publications but not ours. 
Table 2 Univariate Analysis of Relapse in 67 Patients Receiving RTX

\begin{tabular}{|c|c|c|c|}
\hline & Non-Relapse $(n=46)$ & Relapse $(n=2 I)$ & P-value \\
\hline Gender (female/male) & 19/27 & $5 / 16$ & 0.185 \\
\hline Pathology (mcd/fsgs) & $33 / 13$ & $14 / 7$ & 0.775 \\
\hline \multicolumn{4}{|l|}{ Before RTX use } \\
\hline Steroid therapy & 39 (85\%) & 14 (67\%) & 0.112 \\
\hline CTX therapy & $7(15 \%)$ & II (52\%) & 0.003 \\
\hline FK therapy & $16(35 \%)$ & 14 (67\%) & 0.019 \\
\hline Multiple targets therapy & $2(4 \%)$ & $6(29 \%)$ & 0.009 \\
\hline Ciclosporin therapy & 15 (33\%) & 12 (57\%) & 0.067 \\
\hline Disease duration & 36 (IQR: I2-96) & 36 (IQR: 12-96) & 0.19 \\
\hline Relapse time & $3.1 \pm 2.1$ & $4.7 \pm 3.1$ & 0.054 \\
\hline \multicolumn{4}{|l|}{ RTX use } \\
\hline Nephrotic Syndrome (NS) & $19(41 \%)$ & 7 (33\%) & 0.596 \\
\hline Serum creatinine $(\mu \mathrm{mol} / \mathrm{L})$ & $83+56$ & $72+22$ & 0.407 \\
\hline \multicolumn{4}{|c|}{ CDI9+ B-cells (before second RTX course) } \\
\hline CDI9+ B-cells recovery (cells/ul) & $21(46 \%)$ & 15 (7I\%) & 0.034 \\
\hline CD-I 9 count (cells/ul) (IQR) & I $(0-180)$ & $107(12-225)$ & 0.02 \\
\hline RTX consolidation & $31(67 \%)$ & $4(19 \%)$ & $<0.001$ \\
\hline Follow-up (month) (IQR) & $22.5(14,39)$ & $41.5(24.5,52.5)$ & 0.001 \\
\hline
\end{tabular}

Notes: CD-19 count in non-relapsed group: level of CD-19 count before RTX consolidation treatment, or at last follow-up in the non-consolidation group; CD-19 count in relapsed group: the level of CDI9 at the first relapse.

In addition, RTX may help patients maintain a longer period of remission, with fewer side effects. This is regardless of whether they have previously been treated with steroids or/and immunosuppressants. This finding supports previous literature, where high proteinuria level is associated with a worse prognosis of FSGS and MCD. ${ }^{28,29}$ Our study suggests that RTX significantly reduces the number of relapses, and the duration of continuous remission is significantly prolonged, based on the consolidation group with 24-month follow-up. Although some studies suggested that consolidation treatment is unnecessary, citing the absence of, or significant delay

Table 3 Multivariate Cox Regression Analysis of Time to First Relapse

\begin{tabular}{|l|l|l|l|l|}
\hline & P-value & \multicolumn{2}{|l|}{$\begin{array}{l}\text { Exp } \\
\text { (B) }\end{array}$} & \multicolumn{2}{|l|}{$\begin{array}{l}\text { 95\% Exp } \\
\text { (B) Cl }\end{array}$} \\
\hline $\begin{array}{l}\text { Non-consolidation vs consolidation } \\
\text { therapy }\end{array}$ & $<0.001$ & 20.9 & 5.7 & 75.7 \\
\hline $\begin{array}{l}\text { CDI9+ B-cells recovery vs CDI9+ } \\
\text { B-cells non-recovery }\end{array}$ & 0.001 & 6.3 & 2.0 & 19.1 \\
\hline
\end{tabular}

in relapse after CD19+ B-cells recovery, ${ }^{6,30}$ we yielded significantly better results when consolidation therapy was administered. As determined by the Cox model, the risk of relapse was 20 times higher for non-consolidation therapy, and the recovery of CD19+ B-cells is a major risk factor. Given that the recovery of CD19+ B-cells is a major risk factor of relapse $\mathrm{e}^{6,20,31,32}$ and CD19+ B-cells recovery happens at approximately $5-10$ months (average 7.5 months) $)^{7,23,31,33}$ after remission, a consolidation therapy during this time-frame could combat the resurgence of MCD and FSGS, especially for patients with CD19+ B-cells recovery. Our strategy was 1 course of RTX treatment (4 infusions of $375 \mathrm{mg} / \mathrm{m} 2$, every 7-14 days), and a course of RTX consolidation (1-2 infusions of $375 \mathrm{mg} / \mathrm{m} 2) 6$ months after patients were able to achieve and maintain remission. The results we achieved were in line with a RCT of children published in JAMA suggesting that maintenance therapy may decrease relapses of NS for 6 months. ${ }^{23}$

The effectiveness of RTX and its ability to maintain longer remission may lie in its potential mechanisms towards proteinuria that correlates with a worse prognosis 
Table 4 The Baseline Indicators in the Two Groups

\begin{tabular}{|c|c|c|c|}
\hline Characteristic & Non-Consolidation $(n=20)$ & Consolidation $(n=22)$ & P-value \\
\hline Age- $-y r$ & $28 \pm 13$ & $30 \pm 15$ & 0.658 \\
\hline Sex-F/M & $5 / 15$ & $7 / 15$ & 0.738 \\
\hline Renal pathology (MCD/FSGS) & $13 / 7$ & $17 / 5$ & 0.499 \\
\hline \multicolumn{4}{|l|}{ Disease duration before RTX therapy-month } \\
\hline Median & 66 & 48 & \\
\hline Interquartile range & $17-124$ & $24-108$ & \\
\hline History of steroid therapy—no. (\%) & $14(70)$ & $19(86)$ & 0.269 \\
\hline History of CTX therapy-no. (\%) & II (55) & $6(27)$ & 0.115 \\
\hline History of CYA therapy-no. (\%) & $9(45)$ & II (50) & 0.767 \\
\hline History of FK therapy-no. (\%) & $15(75)$ & $8(36)$ & 0.016 \\
\hline History of multiple targets therapy-no. (\%) & $5(25)$ & $3(14)$ & 0.445 \\
\hline Relapse time & $4.6 \pm 2.6$ & $3.3 \pm 2.1$ & 0.097 \\
\hline Creatinine clearance- $-\mathrm{mL} / \mathrm{min} / 1.73 \mathrm{~m} 2$ & $|22 \pm 3|$ & $130 \pm 40$ & 0.454 \\
\hline \multicolumn{4}{|l|}{ Serum albumin-g/l } \\
\hline Average & 29 & 25 & 0.182 \\
\hline Median & 35 & 29 & \\
\hline Interquartile range & $19-39$ & $19-34$ & \\
\hline \multicolumn{4}{|l|}{ Urinary protein $-g / 24 \mathrm{hr}$} \\
\hline Average & 4.47 & 3.66 & 0.351 \\
\hline Median & 1.04 & 1.85 & \\
\hline Interquartile range & $0.11-6.36$ & $0.29-5.52$ & \\
\hline
\end{tabular}

Notes: Forty-two patients who completed the 24-month follow-up were divided into two groups according to whether or not they received consolidation therapy, 20 in non-consolidation group and 22 in consolidation group.

Abbreviations: eGFR, estimated glomerular filtration rate; IQR, interquartile range; CTX, cyclophosphamide; CYA, cyclosporin; FK, tacrolimus.

of MCD and FSGS. ${ }^{28,29}$ RTX acts on CD20, and through tyrosine kinases and mitogen, it activates protein kinases and phospholipase $\mathrm{C} \gamma$, mediating inhibition of B-cell growth or leads to apoptosis. ${ }^{32,34}$ In addition, RTX enhances CTLA4 produced by TREG cells, which in turn inhibits CD80 activation thereby reducing proteinuria. RTX can also reverse the downregulation of SMPDL3b expression and loss of ASMase activity and halts the formation of proteinuria. ${ }^{32}$

The safeness of RTX should also be noted. Amongst patients included in this study, the longest observation time was 5 years. We did not find serious side effects that may be caused by RTX. The most common concerns revolve around infusion reactions and potential side effects such as skin eczema and eosinophilia. These issues were successfully addressed by adopting slow infusion and the use of supportive drugs that combat presenting symptoms.
RTX has also been in clinical use for 25 years, and 15 years for glomerulonephritis. No serious adverse events have been reported. ${ }^{10}$

One concern that patients may have could be the costeffectiveness of RTX. Although consolidation treatment seems to increase the cost of treatment, this factor needs to be weighed against prolonged treatment efficacy, lower relapse rate, and higher quality of life. Our previous studies have compared treatment costs before and after RTX treatment and found that the overall cost may be lower due to a longer remission period. ${ }^{12}$ This study offers no overall comparison of the health economics based on the type of treatment, thus further evidence and a complete list of previous medications are needed to accurately quantify the cost-effectiveness of RTX.

Limitations of this study were that the data are sourced from a single-center covering only the the Asian ethnicity 


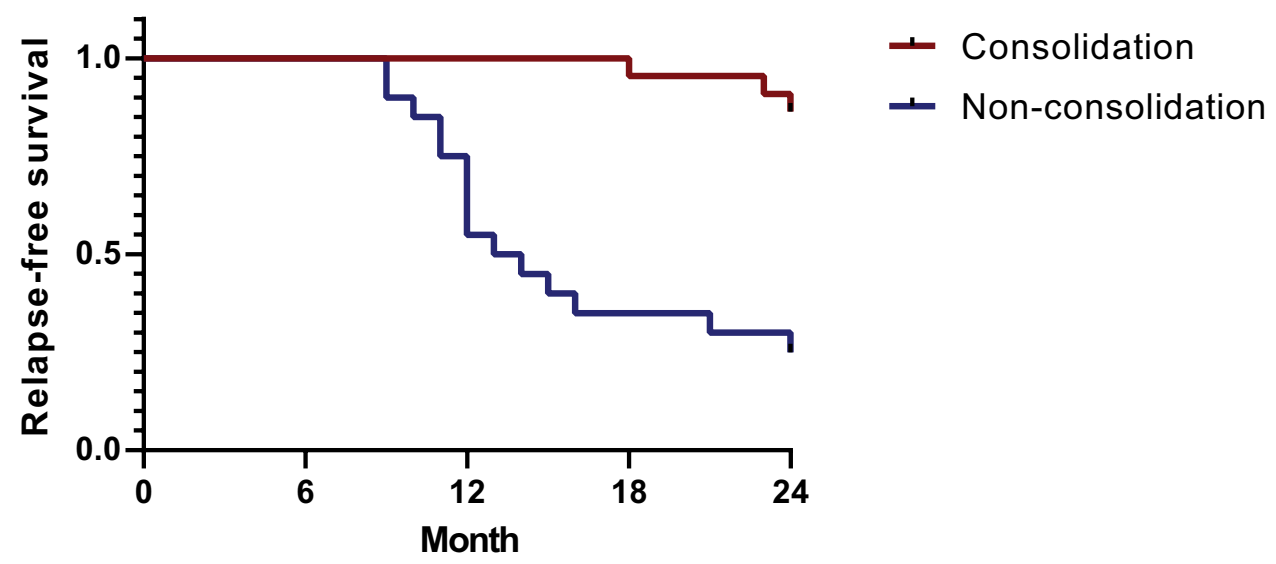

Figure 3 24-month relapse-free survival in the consolidation and non-consolidation groups.

Notes: The 24-month relapse-free survival rate was significantly higher in patients with consolidation compared to non-consolidation (log-rank <0.00I). Only patients who achieved PR or CR and completed 24-month follow-up are included in this graph. The induction RTX was given at the baseline for both groups. Beginning at the 6th month, consolidation treatments were administered for the consolidation group, while non-consolidation groups did not receive further treatment.

and a lack of prospective controlled data. Given that this is a retrospective study and RTX is currently not recommended as a first-line treatment option for MCD and FSGS, there were no data points that could directly compare these approaches using a controlled patient population. Thus, $96 \%$ of our patients have been treated with steroid/immunosuppressants prior to receiving RTX which could potentially create bias. A prospective multi-center controlled study should be conducted so that patients treated only with RTX is available for comparison. In the current study, efforts to minimize selection bias were done by enrolling all eligible patients at our center. In addition, given that the efficacy was calculated based mostly on patients that steroid/immunosuppressive treatment failed to treat, we anticipate that under other conditions RTX would only perform better, for here it is used to treat patients that are harder to treat and thus does not change the validity of our results. Another limitation is that the follow-up time after RTX treatment is shorter than the duration before RTX treatment. To minimize the potential impacts of insufficient follow-up time on the results, we included not only the average number of relapses but also the number of relapses within the first year and the number of relapses per year before and after treatment.

\section{Conclusions}

Given its efficacy, duration of remission, and lesser side effects when compared to steroids and immunosuppression, we believe that RTX may be used as an effective treatment option for FSGS and MCD and that RTX consolidation therapy could be given at 6 months after remission for long-term benefit. However, this study is only observational, and RCT for consolidation therapy are needed.

\section{Acknowledgments}

The authors especially thank all the patients who participated in this study.

\section{Funding}

No funding was received for the conduct of this study or the preparation of this manuscript.

\section{Disclosure}

The authors declare no competing interests.

\section{References}

1. Liu Y, Shi Y, Ren R, Xie J, Wang W, Chen N. Advanced therapeutics in focal and segmental glomerulosclerosis. Nephrology (Carlton). 2018;23(Suppl 4):57-61. doi:10.1111/nep.13463

2. Sinha A, Bagga A. Rituximab therapy in nephrotic syndrome: implications for patients' management. Nat Rev Nephrol. 2013;9(3):154-169. doi:10.1038/nrneph.2012.289

3. Stone JH, Merkel PA, Spiera R, et al. Rituximab versus cyclophosphamide for ANCA-associated vasculitis. $N$ Engl J Med. 2010;363 (3):221-232. doi:10.1056/NEJMoa0909905

4. Jones RB, Furuta S, Tervaert JW, et al. Rituximab versus cyclophosphamide in ANCA-associated renal vasculitis: 2-year results of a randomised trial. Ann Rheum Dis. 2015;74(6):1178-1182. doi:10.1136/annrheumdis-2014-206404

5. Gauckler P, Shin JI, Alberici F, et al. Rituximab in adult minimal change disease and focal segmental glomerulosclerosis - What is known and what is still unknown? Autoimmun Rev. 2020;19 (11):102671. doi:10.1016/j.autrev.2020.102671 
6. Sugiura H, Takei T, Itabashi M, et al. Effect of single-dose rituximab on primary glomerular diseases. Nephron Clin Pract. 2011;117(2): c98-c105. doi:10.1159/000319656

7. Ruggenenti P, Ruggiero B, Cravedi P, et al. Rituximab in steroid-dependent or frequently relapsing idiopathic nephrotic syndrome. J Am Soc Nephrol. 2014;25(4):850-863. doi:10.1681/ ASN.2013030251

8. Roccatello D, Sciascia S, Rossi D, et al. High-dose rituximab ineffective for focal segmental glomerulosclerosis: a long-term observation study. Am J Nephrol. 2017;46(2):108-113. doi:10.1159/000477944

9. Munyentwali H, Bouachi K, Audard V, et al. Rituximab is an efficient and safe treatment in adults with steroid-dependent minimal change disease. Kidney Int. 2013;83(3):511-516. doi:10.1038/ki.2012.444

10. van den Brand J, Ruggenenti P, Chianca A, et al. Safety of rituximab compared with steroids and cyclophosphamide for idiopathic membranous nephropathy. J Am Soc Nephrol. 2017;28(9):2729-2737. doi:10.1681/ASN.2016091022

11. Kronbichler A, Kerschbaum J, Fernandez-Fresnedo G, et al. Rituximab treatment for relapsing minimal change disease and focal segmental glomerulosclerosis: a systematic review. Am J Nephrol. 2014;39(4):322-330. doi:10.1159/000360908

12. Hong ren LL, Shen P, Xiao L, et al. Rituximab treatment in adults with refractory minimal change disease or focal segmental glomerulosclerosis. Oncotarget. 2017;8(55):93438-93443. doi:10.18632/oncotarget.21833

13. Kidney Disease Improving Global Outcomes (KDIGO). KDIGO Clinical Practice Guideline on Glomerular Diseases.https://kdigo. org/wp-content/uploads/2017/02/KDIGO-GN-GL-Public-ReviewDraft_1-June-2020.pdf.

14. Tarshish P, Tobin JN, Bernstein J, Edelmann CM Jr. Prognostic significance of the early course of minimal change nephrotic syndrome: report of the International Study of Kidney Disease in Children. J Am Soc Nephrol. 1997;8(5):769-776.

15. Waldman M, Crew RJ, Valeri A, et al. Adult minimal-change disease: clinical characteristics, treatment, and outcomes. Clin J Am Soc Nephrol. 2007;2(3):445-453. doi:10.2215/CJN.03531006

16. Kyrieleis HA, Lowik MM, Pronk I, et al. Long-term outcome of biopsy-proven, frequently relapsing minimal-change nephrotic syndrome in children. Clin J Am Soc Nephrol. 2009;4(10):1593-1600. doi:10.2215/CJN.05691108

17. Stirling CM, Mathieson P, Boulton-Jones JM, et al. Treatment and outcome of adult patients with primary focal segmental glomerulosclerosis in five UK renal units. QJM. 2005;98(6):443-449. doi:10.1093/qjmed/hci072

18. Francois H, Daugas E, Bensman A, Ronco P. Unexpected efficacy of rituximab in multirelapsing minimal change nephrotic syndrome in the adult: first case report and pathophysiological considerations. $\mathrm{Am}$ J Kidney Dis. 2007;49(1):158-161. doi:10.1053/j.ajkd.2006.10.015

19. Bruchfeld A, Benedek S, Hilderman M, Medin C, Snaedal-Jonsdottir S, Korkeila M. Rituximab for minimal change disease in adults: long-term follow-up. Nephro Dial Transpl. 2013;29(4):851-856. doi:10.1093/ndt/gft312

20. Guitard J, Hebral AL, Fakhouri F, et al. Rituximab for minimal-change nephrotic syndrome in adulthood: predictive factors for response, long-term outcomes and tolerance. Nephrol Dial Transplant. 2014;29 (11):2084-2091. doi:10.1093/ndt/gfu209
21. Iwabuchi Y, Takei T, Moriyama T, Itabashi M, Nitta K. Long-term prognosis of adult patients with steroid-dependent minimal change nephrotic syndrome following rituximab treatment. Medicine (Baltimore). 2014;93(29):e300. doi:10.1097/MD.0000000000000300

22. DaSilva I, Huerta A, Quintana L, et al. Rituximab for steroid-dependent or frequently relapsing idiopathic nephrotic syndrome in adults: a retrospective, Multicenter Study in Spain. BioDrugs. 2017;31(3):239-249. doi:10.1007/s40259-017-0221-x

23. Basu B, Sander A, Roy B, et al. Efficacy of rituximab vs tacrolimus in pediatric corticosteroid-dependent nephrotic syndrome: a Randomized Clinical Trial. JAMA Pediatr. 2018;172(8):757-764. doi:10.1001/jamapediatrics.2018.1323

24. Cravedi P, Remuzzi G, Ruggenenti P. Rituximab in primary membranous nephropathy: first-line therapy, why not? Nephron Clin Pract. 2014;128(3-4):261-269. doi:10.1159/000368589

25. Ruggenenti P, Fervenza FC, Remuzzi G. Treatment of membranous nephropathy: time for a paradigm shift. Nat Rev Nephrol. 2017;13 (9):563-579. doi:10.1038/nrneph.2017.92

26. Fervenza FC, Appel GB, Barbour SJ, et al. Rituximab or cyclosporine in the treatment of membranous nephropathy. $N$ Engl J Med. 2019;381(1):36-46. doi:10.1056/NEJMoa1814427

27. Hansrivijit P, Cheungpasitporn W, Thongprayoon C, Ghahramani N Rituximab therapy for focal segmental glomerulosclerosis and minimal change disease in adults: a systematic review and meta-analysis. BMC Nephrol. 2020;21(1):134. doi:10.1186/s12882-020-01797-7

28. Zoja C, Benigni A, Remuzzi G. Cellular responses to protein overload: key event in renal disease progression. Curr Opin Nephrol Hypertens. 2004;13(1):31-37. doi:10.1097/00041552-20040100000005

29. Peired A, Angelotti ML, Ronconi E, et al. Proteinuria impairs podocyte regeneration by sequestering retinoic acid. J Am Soc Nephrol. 2013;24(11):1756-1768. doi:10.1681/ASN.2012090950

30. Takei T, Itabashi M, Moriyama T, et al. Effect of single-dose rituximab on steroid-dependent minimal-change nephrotic syndrome in adults. Nephrol Dial Transplant. 2013;28(5):1225-1232. doi:10.1093/ndt/gfs515

31. Katsuno T, Masuda T, Saito S, et al. Therapeutic efficacy of rituximab for the management of adult-onset steroid-dependent nephrotic syndrome: a retrospective study. Clin Exp Nephrol. 2019;23 (2):207-214. doi:10.1007/s10157-018-1630-y

32. Fornoni A, Sageshima J, Wei C, et al. Rituximab targets podocytes in recurrent focal segmental glomerulosclerosis. Sci Transl Med. 2011;3 (85):85ra46. doi:10.1126/scitranslmed.3002231

33. Wang X, Cui Z, Zhang YM, et al. Rituximab for non-responsive idiopathic membranous nephropathy in a Chinese cohort. Nephrol Dial Transplant. 2018;33(9):1558-1563.

34. Tokunaga M, Fujii K, Saito K, et al. Down-regulation of CD40 and CD80 on B cells in patients with life-threatening systemic lupus erythematosus after successful treatment with rituximab. Rheumatology (Oxford). 2005;44(2):176-182. doi:10.1093/rheumatology/keh443

\section{Publish your work in this journal}

Drug Design, Development and Therapy is an international, peerreviewed open-access journal that spans the spectrum of drug design and development through to clinical applications. Clinical outcomes, patient safety, and programs for the development and effective, safe, and sustained use of medicines are a feature of the journal, which has also been accepted for indexing on PubMed Central. The manuscript management system is completely online and includes a very quick and fair peer-review system, which is all easy to use. Visit http://www. dovepress.com/testimonials.php to read real quotes from published authors. 